\title{
Cranial Defect Overlying a Ventriculoperitoneal Shunt: Pressure Gradient Leading to Free Flap Deterioration?
}

\author{
Jae Doo Joo', \\ Jin-Uk Jang ${ }^{2}$, \\ Hyonsurk Kim¹, \\ Eul-Sik Yoon', \\ Dong Hee Kang ${ }^{1}$ \\ ${ }^{1}$ Department of Plastic and Reconstructive \\ Surgery, Dankook University College of \\ Medicine, Cheonan; \\ ${ }^{2}$ Department of Plastic and Reconstructive \\ Surgery, Korea University Anam Hospital, \\ Korea University College of Medicine, Seoul, \\ Korea
}

No potential conflict of interest relevant to this article was reported.

\begin{abstract}
We report a case of free flap deterioration which may have been induced by pressure gradient resulting from cranial defect overlying a ventriculoperitoneal shunt (VP shunt). The patient, male and aged 78, had a VP shunt operation for progressive hydrocephalus. Afterwards, the scalp skin flap surrounding the VP shunt collapsed and showed signs of necrosis, exposing part of the shunt catheter. After covering the defect with a radial forearm free flap, the free flap site showed signs of gradual sinking while the vascularity of the flap remained unimpaired. An agreement was reached to remove the shunt device and observe the patient for any neurological symptoms, and after the shunt was removed and the previous cranial opening filled with fibrin glue by Neurosurgery, we debrided the deteriorated flap and provided coverage with 2 large opposing rotational flaps. During 2 months' outpatient follow-up no neurological symptoms appeared, and the new scalp flap displayed slight depression but remained intact. The patient has declined from any further follow-up since.
\end{abstract}

Keywords: Decompressive craniectomy / Free tissue flaps / Ventriculoperitoneal shunt

\section{INTRODUCTION}

Reconstruction after craniofacial fractures, tumor resection, and decompression of intracranial hemorrhages frequently requires cooperation between the fields of plastic surgery and neurosurgery. Reconstruction relating to craniofacial wound problems or scalp defects occurring after primary neurosurgery also commonly requires the help of plastic surgeons. The low elasticity and robust blood flow of scalp tissues are taken into account when reconstructing soft tissue defects in the scalp area [1]; accordingly, plastic surgeons can choose from several surgical method options including large local flaps, staged operations using tissue expanders, and free flaps, each having inherent pros and cons. This study

\section{Correspondence: Hyonsurk Kim}

Department of Plastic and Reconstructive Surgery, Dankook University Hospital, Dankook University College of Medicine, 201 Manghyang-ro, Dongnam-gu, Cheonan 31116, Korea

E-mail: kruezel@gmail.com

Received March 15, 2017 / Revised August 7, 2017 / Accepted August 9, 2017 reports on a case where a free flap used to cover a ventriculoperitoneal shunt (VP shunt) inserted for treatment of post-cerebral hemorrhage hydrocephalus gradually sunk and deteriorated.

\section{CASE REPORT}

The patient, male and aged 78, had received coil embolization in 2006 for subarachnoid hemorrhage caused by a ruptured basilar tip aneurysm, and subsequently had a VP shunt inserted in February 2012 due to progressive hydrocephalus (Fig. 1). Afterwards, the patient's progress seemed satisfactory until April 2015, when the scalp skin flap surrounding the VP shunt collapsed and showed signs of necrosis, exposing part of the shunt catheter (Fig. 2). The attending neurosurgeon, focusing only on skin necrosis and not on flap retraction or collapse, attempted to revise the wound by debriding the area, inserting a plate to support the skin flap overlying the shunt, and repairing; when the repair site displayed signs 
of necrosis again within 2 weeks the case was referred to the Department of Plastic Surgery, albeit without any mention of the original flap collapsing and only stating hardware exposure and flap necrosis. On visual inspection, the recently-inserted metal plate was exposed as the scalp area surrounding the repair site grew thinner. Total debridement of all necrotic and unstable portions resulted in a $5 \times 4 \mathrm{~cm}$ defect, and as we had no prior knowledge of flap sinkage and thinking the patient to be a rather simple case of infection or hardware exposure, we covered the defect with a radial forearm free flap elevated from the patient's left arm with

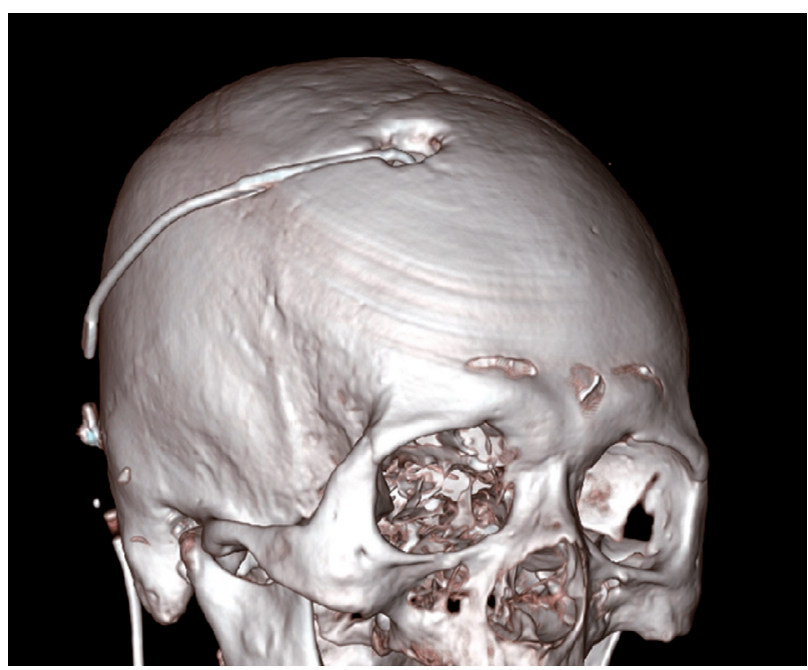

Fig. 1. Ventriculoperitoneal shunt (VP). Initial computed tomography scan, showing the results after partial craniectomy for VP shunt insertion.

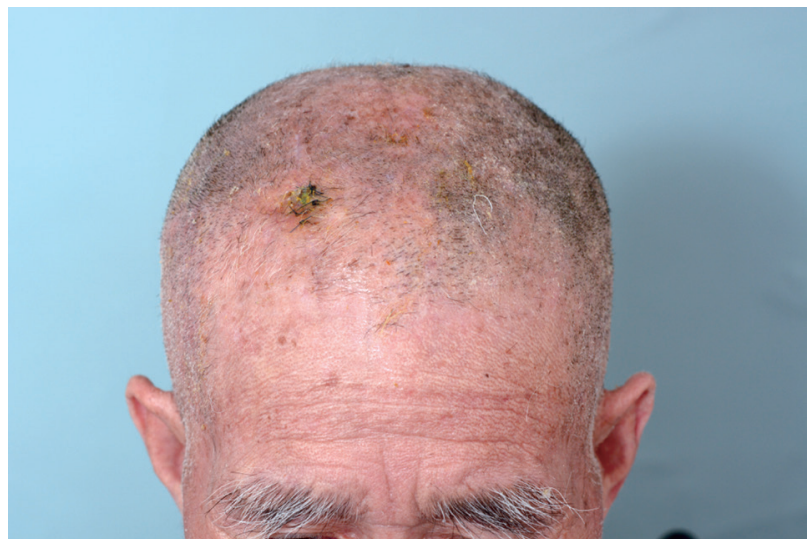

Fig. 2. Initially referred patient status. Clinical photo taken when the case was first referred to our Department, displaying partial exposure of the plate inserted for prevention of scalp collapse. the right-sided superficial temporal artery as a recipient vessel, judging that the free flap's robust blood supply would prevent infection and necrosis (Fig. 3). Since the Neurosurgery department had already conducted several surgical procedures on the patient, free flap coverage was considered to be a better choice than a simple local flap operation anticipating the possibility of compromised scalp flap viability and adhesion to underlying bone. The thin radial forearm flap was chosen over a muscle flap to reduce operative time, considering the patient's old age, and for superior scalp contour. After free flap coverage, the patient's postoperative course was uneventful, and was lost to follow-up after 3 weeks, ignoring outpatient clinic appointments. However, after 4 months, the patient visited our outpatient clinic stating that the inserted
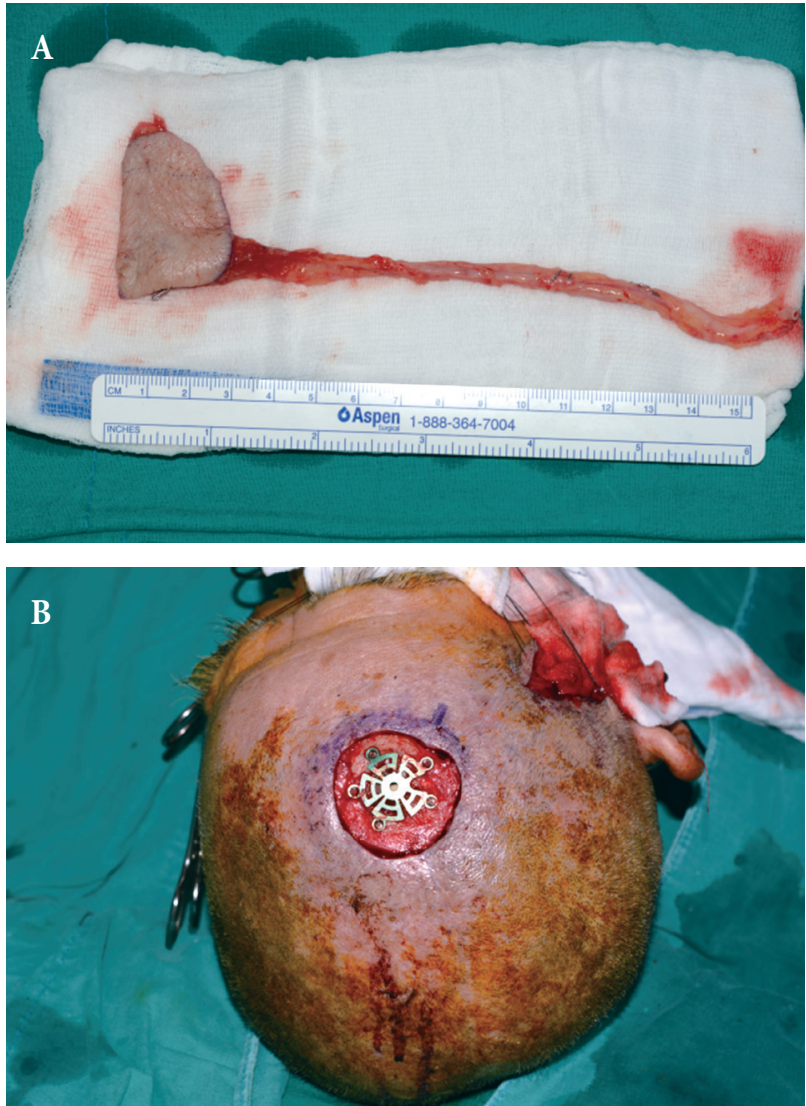

Fig. 3. First reconstruction using radial forearm free flap. Intraoperative photograph of (A) $5 \times 4 \mathrm{~cm}$ radial forearm free flap elevated from left arm to cover (B) post-debridement scalp defect. Unfortunately, the patient was lost from follow-up after discharge until presenting with flap deterioration several months later, precluding any visual documentation of the stable postoperative state. 
plate was again visible (Fig. 4). The free flap site was showing signs of gradual sinking while the vascularity of the flap remained unimpaired, with clear arterial sounds audible with handheld Doppler probing. The flap covering the hardware had grown substantially thinner and appeared as if a skin graft had been directly applied to the plate. No signs of infection were detected. There was also no evidence suggesting disorders of collagen metabolism such as nutritional deficiency, malignancy or liver cirrhosis that could lead to flap thinning and weakening [2]. Accordingly, we discussed with the Neurosurgery department the possibility of the VP shunt creating a constant negative pressure gradient on the overlying flap leading to its collapse, and the options of re-locating the shunt for pressure adjustment. An agreement was reached to remove the shunt device and observe the patient for any neurological symptoms, and after the shunt and covering metal plate were removed and the previous cranial opening filled with fibrin glue by Neurosurgery, we debrided the deteriorated flap and provided coverage with 2 large opposing rotational flaps. Considering the patient's old age, the possibility of recurrent flap sinking and collapse made a second free flap transfer procedure seem too risky, and the smaller size of the new post-debridement defect allowed local flap coverage without previous scarring and adhesion causing much trouble. Both the patient and the surgical site recovered as expected with no complications until discharge,

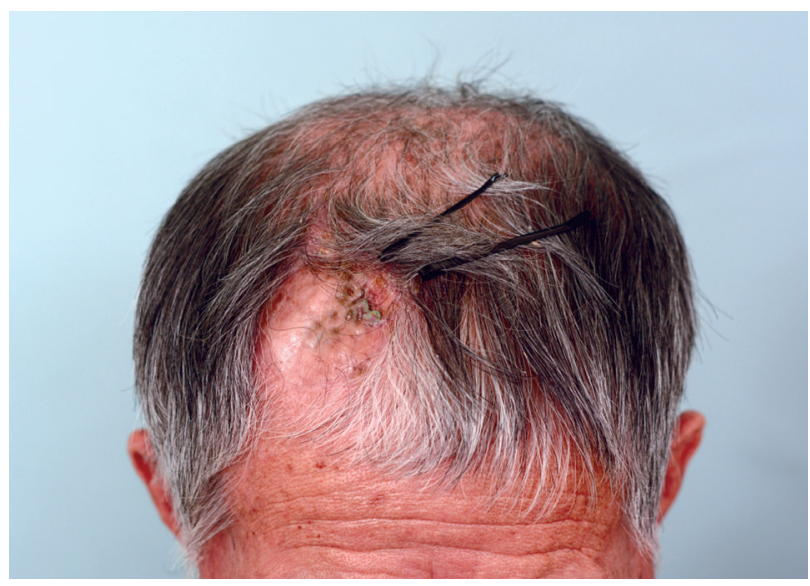

Fig. 4. Sinking flap syndrome. Follow up photo taken 4 months after free flap reconstruction. Although no tension of surrounding tissue or signs of infections were observed, the flap has almost sunken into the plate and shunt area with hardware exposure. and during 2 months' outpatient follow-up no neurological symptoms appeared, the scalp flap displayed slight depression, but remained in good condition (Fig. 5); the patient has declined from any further follow-up since.

\section{DISCUSSION}

'Sinking flap syndrome' is a term we have newly coined. This term refers to the phenomenon of incremental flap retraction into underlying tissue as if melting down, failing to cover deep tissue (bone, dura mater, etc.), which may be attributable to constant pressure gradient force between the two sides of the flap. As the metal plate was thin and fixed firmly to the cranium, and the free flap allowed tensionless, stable tissue coverage with firm durability, spontaneous hardware exposure as a common postoperative complication was ruled out. While the term 'sinking skin flap syndrome' has been used to describe neurologic symptoms related to scalp sinking and brain herniation after wide decompressive craniectomy, the terminology was not applicable to this case as it focuses mainly on the neurologic symptoms observed, rather than on wound problems [3-5]. A number of cases have been reported on covering VP shunts (due to ischemic damage resulting from tension and circulation issues) [6], but the present case is unprecedented as it reports an observation of chronic flap sinking

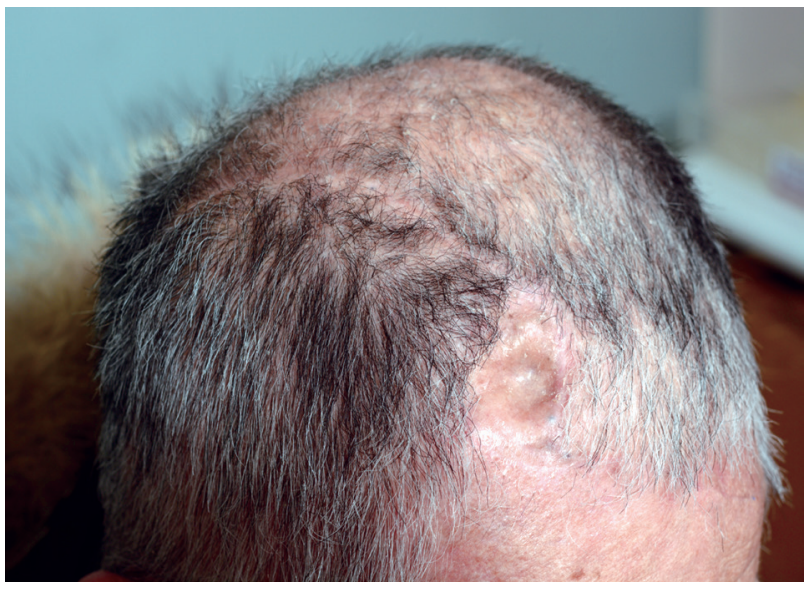

Fig. 5. Postoperative 2 months image after second reconstruction. The scalp flap displayed some recurrent sinking, albeit without evidence of internal tissue exposure such as dura, cranium, etc. 
after successful free flap coverage over VP shunt site without any acute phase complications. A literature review failed to find any case reports or original articles with close resemblance, or corresponding, to this case; therefore, explaining the pathophysiology of this case had limitations. The fact that initial sinkage and necrosis of the scalp flap overlying the VP shunt only occurred 3 years after shunt insertion implies that intracranial or intracerebral changes may have somehow induced flap collapse. No previous studies, animal or human, have been published regarding how cranial defects affect the balance of atmospheric and intracranial pressure, and what size of defects leads to symptoms. Basically, as the cranium acts as a 'shield' protecting intracranial contents, it could be hypothesized that it also protects the cerebral tissue from atmospheric pressure when intracranial pressure is lower. The metal plate used to cover the cranial defect in this case was not a solid barrier and had several fenestration holes through which the pressure difference could be transferred, and evidently through which the free flap gradually deteriorated. In 'sinking skin flap syndrome', which seems to share a common 'post-craniectomy difference between atmospheric and intracranial pressure' pathophysiology with this case, direct transmission of atmospheric pressure is considered as the main cause [3]. The observation that temporarily increasing intracranial pressure by placing the patient in the Trendelenburg position may improve the patient's neurological condition also points in the same direction [7]. Han et al. [8] have also reported that, "after VP shunt, the increased negative pressure gradient between atmospheric and intracranial pressure may have caused neurological deterioration such as the syndrome of the sinking skin flap", noting that VP shunt may attribute to increased pressure gradient. While flap necrosis or wound dehiscence due to poor circulation resulting from constant pressure in free flaps can occur, to the best of our knowledge this is the first report of such a complication relating to negative pressure gradient and chronic sinking. The small size of the cranial defect may have limited the effect of the negative pressure gradient on intracranial contents (and ensuing neurological symptoms), instead focalizing into flap deterioration. The firm metal plate underlying the sinking flap probably would have applied greater pressure to the flap undersurface compared to more pliable dura, therefor acting as another factor in flap deterioration; the presence of this fenestrated metal plate indeed may be the main difference between this case and other cases of 'sinking skin flap syndrome', where the scalp flap sinks but necrosis does not occur.

It appears that further research is necessary to understand how much negative pressure gradients cause substantial flap sinking or melting, how large a defect can cause neurological symptoms, and whether such defects can cause local wound problems without neurological symptoms. Applying hardware or tissue that can act as a solid barrier against the pressure gradient (instead of mesh-like plates filled with holes, which led to flap sinking in this case) may be a viable option and should be considered in flap coverage of similar cases, along with removal of any unnecessary foreign material which may cause irritation or tension. While the VP shunt was removed in this case, we believe that complications may be reduced through VP shunt device pressure adjustment [8]. To conclude, when conducting craniofacial reconstruction, the possibility of 'sinking skin flap syndrome' or 'sinking flap syndrome' must be taken into account, both being complications resulting from atmospheric and intracranial pressure gradients after craniectomy.

\section{PATIENT CONSENT}

The patient provided written informed consent for the publication and the use of his images.

\section{REFERENCES}

1. Desai SC, Sand JP, Sharon JD, Branham G, Nussenbaum B. Scalp reconstruction: an algorithmic approach and systematic review. JAMA Facial Plast Surg 2015;17:56-66.

2. Minor RR. Collagen metabolism: a comparison of diseases of collagen and diseases affecting collagen. Am J Pathol 1980;98:225-80.

3. Annan M, De Toffol B, Hommet C, Mondon K. Sinking skin flap syndrome (or Syndrome of the trephined): A review. Br J Neurosurg 2015;29:314-8.

4. Yamaura A, Makino H. Neurological deficits in the presence of the sinking skin flap following decompressive craniectomy. Neurol Med Chir (Tokyo) 1977;17:43-53. 
5. Stula D. The problem of the "sinking skin-flap syndrome" in cranioplasty. J Maxillofac Surg 1982;10:142-5.

6. Nguyen TA, Cohen PR. Scalp necrosis overlying a ventriculoperitoneal shunt: a case report and literature review. Dermatol Online J 2015;21.

7. Chalouhi N, Teufack S, Fernando Gonzalez L, Rosenwasser RH, Jab- bour PM. An extreme case of the syndrome of the trephined requiring the use of a novel titanium plate. Neurologist 2012;18:423-5.

8. Han PY, Kim JH, Kang HI, Kim JS. "Syndrome of the sinking skinflap" secondary to the ventriculoperitoneal shunt after craniectomy. J Korean Neurosurg Soc 2008;43:51-3. 